\title{
RETROSPECTIVE ANALYSIS OF USE OF EMPIRIC ANTI TUBERCULOSIS TREATMENT IN SMEAR NEGATIVE PULMONARY TUBERCULOSIS PATIENTS IN CENTRAL SRI LANKA
}

\author{
Madegedara $D^{1}$, Kulathunga K M C N ${ }^{2}$, Nakandala S C \\ ${ }^{1}$ Consultant Respiratory Physician, Respiratory Disease Treatment Unit, Teaching Hospital, Kandy, Sri Lanka \\ ${ }^{2}$ Senior Registrar, Respiratory Disease Treatment Unit, Teaching Hospital, Kandy, Sri Lanka
}

\begin{abstract}
Objective: To critically analyze the outcome of sputum negative pulmonary TB patients who were managed in the Respiratory unit, Kandy Sri Lanka.

Setting: Respiratory Unit, Teaching hospital, Kandy.

Method: The subjects were between age 12 years or older and visited chest clinic Kandy, between January -December 2008 with a suspicion of pulmonary TB. 84 patients were reviewed with admission note, microbiology results and chest radiography. The characteristics of patients such as age, sex, clinical features, laboratory and radiographic findings were analyzed.
\end{abstract}

Results: The diagnosis of definite or probable smear negative pulmonary TB was made on treatment outcome at one month with ATT medication. At this endpoint, 67 (79.76\%) had clinical and radiological improvement, $7(8.33 \%)$ had clinical improvement and $3(3.57 \%)$ had radiological improvement. Mantoux test was positive in $46(78 \%)$ of definite cases and $6(85.7 \%)$ in probable cases. Mycobacterial culture was positive in 5 patients. $7(8.3 \%)$ cases were diagnosed as 'non-TB' based on absence of clinical / radiological improvement or discovery of another cause at or before this study end point. In non TB group 3 had carcinoma, 2 had pneumoconiosis, 1 had bronchiectasis while in 1, cause was indeterminate.

Mantoux became positive in $6(83.3 \%)$ in non Tuberculosis group. There was no association between positive Mantoux and presence of active pulmonary TB.

Conclusion: Our findings suggest that empirical anti TB treatment is an acceptable practice if clinical suspicion of tuberculosis is high and patients show supportive radiological evidence of active TB.

Keywords: Smear negative pulmonary tuberculosis, empirical anti tubercular treatment

\section{Correspondence:}

Dr. Dushantha Madegedara

MBBS,MD, FCCP(USA), FCCP(SL),FRCP(Edin)

Consultant Respiratory Physician

Teaching Hospital, Kandy

Sri Lanka

E-mail: dmadegedara@yahoo.com

Tel/Fax: 0094812384374

\section{INTRODUCTION}

Mycobacterium Tuberculosis (MTB) infects over one third of the world's population, causes 8 million new cases of disease, and over 2 million deaths every year worldwide. ${ }^{1}$ Tuberculosis (TB) mainly affects people in the economically active age groups which results in an immense loss to communities and countries.

The WHO south East Asia region carries the highest burden of tuberculosis among all WHO regions. 
Although Sri Lanka is not among the high burden countries, TB remains a widespread problem and poses a continuing threat to the health and development of the people in the country. ${ }^{2}$

Nearly 17000 people (80/100 000) are currently estimated to be suffering from tuberculosis. Every year, it's estimated that more than 11000 new cases (60/100 000) arise. Over 50000 people are expected to develop TB disease during next 5 years and out of this only half are expected to be smear positive TB cases. $^{3}$

The confirmation of TB diagnosis is made by the detection of MTB bacilli. The detection of acid fast bacilli (AFB) on smear of respiratory specimensputum, induced sputum or bronchial washing, is essential for diagnosis of pulmonary TB. ${ }^{4}$

However sensitivity of the AFB smear result is known to be poor, varying between $30 \%-70 \%$ depending on the number of factors relating to how the test is being performed.

Although the culture of the tuberculosis bacilli is more sensitive ( $80 \%-85 \%)$, being able to detect as few as 10 bacteria per milliliter of sputum, it usually takes 3-6 weeks to receive culture results.

However considering the fact that nearly half of all patients with TB are smear negative, decision to start anti-TB treatment in patients with a high suspicion of TB can only be made on clinical scenario and typical radiological feature that not only benefits the patients, but also helps to control TB in the community. 5,6

In Sri Lanka despite an effective national programme for tuberculosis control and mass scale immunoprophylaxis, tuberculosis still remains a growing public health issue with over 9000 new cases being detected annually. ${ }^{7}$

The empirical treatment of presumptive pulmonary TB is especially important in Sri Lanka where the prevalence of pulmonary TB is still high and only one third of the patients with pulmonary TB are sputum AFB smear positive., $, 9,10$ The objective of this study was to critically analyze the outcome of sputum negative pulmonary TB patients who were managed in the chest clinic, Kandy.

\section{METHODOLOGY}

We assessed the 84 patients who were registered as smear negative TB patients in chest clinic Kandy. The patients were of 12 years of age and above. The study was conducted over 12 month period January to December 2008.

These patients were retrospectively reviewed using the clinical records and chest radiographs. All of them had 3 negative sputum AFB smear results and started anti TB medication by individual physician's decision on the basis of symptoms and or radiographic features. All sputum smears were examined by trained microbiology technicians.

Medical records of these patients reviewed which includedanadmissionnote, microbiology results(smear and culture) and chest radiography interpretation. Data were collected using a questionnaire. We analyzed demographic characteristic of patients such as age and sex as well as clinical features including symptoms, laboratory and radiographic findings.

The patients were categorized as definite and probable cases depending on presence of both clinical and radiological improvement as evidence (definite) and only either one of it. (Probable)

\section{RESULTS}

Eighty four patients who were treated as smear negative TB between January-December 2008 were analyzed. The mean age of the patient was 48 years with a male to female ratio was 61:39. Figure 1.

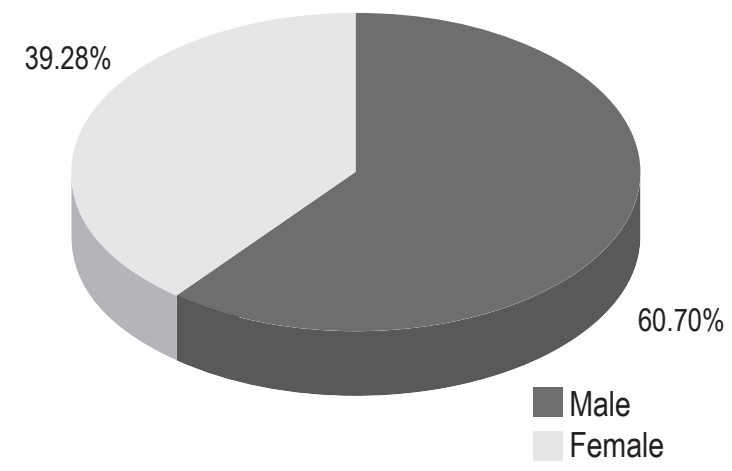

Fig. 1. Sex distribution of Study Population 
The mean duration of treatment was 6 months 67 patients $(79.76 \%)$ had both clinical and radiological improvement (definite cases), 7 patients (8.33\%) had clinical improvement alone (probable cases) and $3(3.57 \%)$ had radiological improvement alone (probable cases) figure 2.

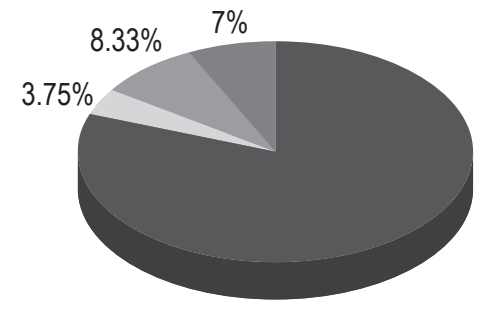

Definit cases

Radiological Improvement only (Probably)

Clinical Improvement only (Probably)

non TB Group

$79.75 \%$

Fig. 2. Treatment outcome at 2 Months

Of the definite group $46(78 \%)$ had strongly positive Mantoux test and in probable group it's 6 (85.7\%). Figure 3.

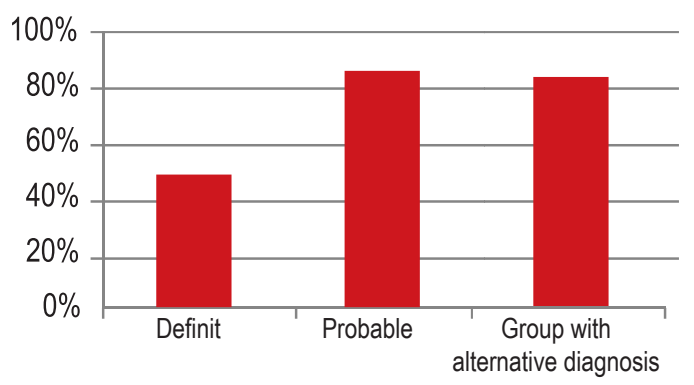

Fig: 3. Mantoux Positivity in Different Treatment

Confirmation of acid fast bacilli was subsequently obtained in 5 patients $(7.4 \%)$ (all definite cases) from culture of initial pulmonary specimens. Seven patients (8.3\%) were diagnosed as non TB based on absence of both clinical and radiological improvement or discovery of another cause for the pulmonary condition at or before this 2 month study end point. In non TB group 3 had carcinoma, Two patients had pneumoconiosis one had bronchiectasis while in 1 patient cause was indeterminate.

12 patients of this group showed adverse effects due to TB medication such as hepatitis, pruritis and however they were not so severe as to change or stop anti TB medications.

\section{DISCUSSION}

The prompt initiation of treatment for pulmonary TB is the crucial factor which is responsible for controlling disease spread in the community. The fact that the smear negative but culture positive TB was responsible for the $17 \%$ of the TB transmission highlight the importance of initiation of anti TB medication in suspected cases of smear negative pulmonary TB at appropriate time.., 9

The definitive diagnosis of TB needs either confirmation by sputum positivity or culture positivity. However both these depend on various factors such as patient cooperation lab facility availability, culture media availability etc. Although newer rapid diagnostic tools such as nucleic acid amplication method and serodiagnosis using ELISA have been introduced recently they are not yet considered as standard practice. . $, 6,7$

In analysis of our study we found that decision to treat patients empirically with anti TB medications on selective basis is appropriate, as $91.63 \%$ showed improvement. (Both definite and probable group) But positive culture for AFB was noted only in $6.4 \%$ of this group which is far less than expected. This could be attributed to multiple factors related to patients and as well as technical problems associated with culturing AFB. ${ }^{11}$

However positivity of Mantoux test showed no significant difference among these two groups, as both these groups show more than $78 \%$ positivity. This fact needs further evaluation as we have to continue to follow up them further to see whether they too will develop TB or not.

There are no similar studies done in Sri Lanka to assess the outcome of patients who were treated as smear negative pulmonary TB. This study showed that use of appropriate criteria's to start treatment in selected groups (definite/probable) are worthwhile practice. 
It should be taken in to consideration that these groups need very careful follow up in frequent intervals. One should look for clinical and radiological improvement in these selected groups and if there's no improvement there's need to look for possible drug resistant or secondary causes like connective tissue diseases, bronchial malignancy etc which can mimic pulmonary Tuberculosis.

\section{Limitations of this study}

The short coming of this analysis was the fact we were unable to document clearly the criteria which were taken as clinical improvement and monitor these patients closely as this was retrospective study. In addition there were practical problems of collection, transport and culturing specimens. This may have contributed to poor outcome of culture results in this study.

\section{REFERENCES}

1. Dolin PJ, Raviglinone $M C$, and Kochi A: Global tuberculosis incidence and mortality during 19902000. Bull world health organ 1994:72:213-220.

2. National programme for tuberculosis control and chest disease. General manual for tuberculosis control. $2^{\text {nd }}$ ed. Ministry of health, srilanka: 2005

3. Guidelines for Management of Tuberculosis on children. National Programme for Tuberculosis control and Chest Diseases. Ministry of Health, Srilanaka, January 2008.
4. Foulds J, O'Brian R: new tools for the diagnosis of tuberculosis: the perspective of developing countries. Int $j$ tuberc lung dis 1998:2:778-783.

5. Gordin FM.Slutkin G,Schecter G,Goodman PC,Hopewell PC:Presumptive diagnosis and treatment of pulmonary tuberculosis based on radiographic findings. Am Rev Respir Dis1989;139:1090-1093.

6. Woodring $\mathrm{JH}$,Vandiviere $\mathrm{HM}$,Fried AM,Dillon ML,Williams TD,Melvin IG:Update:The radiographic features of pulmonary tuberculosis.AJR Am J Roentgenol 1986;146:497-506.

7. Behr MA.Warren SA, Salamon H, Hopewell PC, Ponce de Leon A, Daley CL, Small PM: Transmission of Mycobacterium tuberculosis from patients smear negative for acid fast bacilli. Lancet 1999:353:444449

8. Whqlibdoc.who.int/hq / 2003 / WHO - CDS - TB -2003.315.pdf

9. Siddiqi K,Lambert M,Wally J.Clinical diagnosis of smear-negative pulmonary tuberculosis in low-income countries:the current evidence. The Lancet Infectious Diseases 2003;3:5:288-296.

10. Colebunders R,Bastien I.A review of the diagnosis and treatment of smear-negative pulmonary tuberculosis. Int J Tuberc Lung Dis 2000;4:97-107.

11. Anglaret $X$, Saba J,Perronne C,Lacassin F,Longuet P,Leport C,Vilde JL:Empiric antituberculosis treatment:Benefits for earlier diagnosis and treatment of tuberculosis.Tuber Lung Dis 1994;75:334-340. 